\title{
Robust stability of differential-algebraic equations
}

\author{
Nguyen Huu Du and Vu Hoang Linh* and Volker Mehrmann ${ }^{\dagger}$
}

\begin{abstract}
This paper presents a survey of recent results on the robust stability analysis and the distance to instability for linear time-invariant and time-varying differential-algebraic equations (DAEs). Different stability concepts such as exponential and asymptotic stability are studied and their robustness is analyzed under general as well as restricted sets of real or complex perturbations. Formulas for the distances are presented whenever these are available and the continuity of the distances in terms of the data is discussed. Some open problems and challenges are indicated.
\end{abstract}

Keywords: differential-algebraic equation, robust stability, stability radius, spectrum, spectral set, restricted perturbation.

AMS(MOS) subject classification: 93B35, 93D09, 34A09, 34D10

\section{Introduction}

In many areas of science and engineering one uses mathematical models to simulate, control or optimize a system or process. These mathematical models, however, are typically inexact or contain uncertainties and thus, the following question is of major importance.

How robust is a specific property of a given system described by differential or difference equations under perturbations to the data?

Here, we say that a certain property of a system is robust if it is preserved when an arbitrary (but sufficiently small) perturbation affects the system. An important quantity in this respect is then the distance (measured by an appropriate metric) between the nominal system and the closest perturbed system that does not possess the mentioned property, this is typically called the radius of the system property.

\footnotetext{
Nguyen Huu Du

Faculty of Mathematics, Mechanics and Informatics, Vietnam National University, 334, Nguyen Trai Str., Thanh Xuan, Hanoi, Vietnam e-mail: dunhevnu.edu.vn

Vu Hoang Linh

Faculty of Mathematics, Mechanics and Informatics, Vietnam National University, 334, Nguyen Trai Str., Thanh Xuan, Hanoi, Vietnam e-mail: linhvh@vnu.edu.vn

Volker Mehrmann

Institut für Mathematik, MA 4-5, Technische Universität Berlin, D-10623 Berlin, Fed. Rep. Germany e-mail: mehrmann@ math.tu-berlin.de

* Supported by NAFOSTED Grant 101.01-2011.14.

$\dagger$ Supported by Deutsche Forschungsgemeinschaft, through project A02 within Collaborative Research Center 910 Control of self-organizing nonlinear systems
} 
In this paper, we deal with robustness and distance problems for differential-algebraic equations (DAEs), with a focus on robust stability and stability radii. Systems of DAEs, which are also called descriptor systems in the control literature, are a very convenient modeling concept in various real-life applications such as mechanical multibody systems, electrical circuit simulation, chemical reactions, semi-discretized partial differential equations, and in general for automatically generated coupled systems, see [7, 33, 41, $56,60,76,77]$ and the references therein.

DAEs are generalizations of ordinary differential equations (ODEs) in that certain algebraic equations constrain the dynamical behavior. Since the dynamics of DAEs is constrained to a set which often is only given implicitly, many theoretical and numerical difficulties arise, which may lead to a sensitive behavior of the solution of DAEs to perturbation in the data. The difficulties are characterized by fundamental notions for DAEs such as regularity, index, solution subspace, or hidden constraints, which do not arise for ODEs. These properties may be easily lost when the data are subject to arbitrarily small perturbations. As a consequence, usually restrictions to the allowed perturbations have to be made, leading to robustness questions for DAEs that are very different from those for ODEs.

This paper surveys robustness results for linear DAEs with time-invariant or time-varying coefficients of the form

$$
E \dot{x}=A x+f,
$$

on the half-line $\mathbb{I}=[0, \infty)$, together with an initial condition

$$
x\left(t_{0}\right)=x_{0}, t_{0} \in \mathbb{I} .
$$

Here we assume that $E, A \in C\left(\mathbb{I}, \mathbb{K}^{n \times n}\right)$, and $f \in C\left(\mathbb{I}, \mathbb{K}^{n}\right)$ are sufficiently smooth. We use the notation $C\left(\mathbb{I}, \mathbb{K}^{n \times n}\right)$ to denote the space of continuous functions from $\mathbb{I}$ to $\mathbb{K}^{n \times n}$, where $\mathbb{K}=\mathbb{R}$ or $\mathbb{K}=\mathbb{C}$.

Linear systems of the form (1) arise directly in many applications and via linearization around solution trajectories [16]. They describe the local behavior in the neighborhood of a solution for general implicit nonlinear system of DAEs

$$
F(t, x, \dot{x})=0,
$$

the constant coefficient case arising in the case of linearization around stationary solutions.

Recall the following classical stability concepts for ordinary differential equations

$$
\dot{x}=f(t, x), \quad t \in \mathbb{I},
$$

with initial condition (2), see e.g. [51].

Definition 1. A solution $x: t \mapsto x\left(t ; t_{0}, x_{0}\right)$ of (4) with initial condition (2) is called

1. stable if for every $\varepsilon>0$ there exists $\delta>0$ such that

a. the initial value problem (4) with initial condition $x\left(t_{0}\right)=\hat{x}_{0}$ is solvable on $\mathbb{I}$ for all $\hat{x}_{0} \in \mathbb{K}^{n}$ with $\left\|\hat{x}_{0}-x_{0}\right\|<\delta$

b. the solution $x\left(t ; t_{0}, \hat{x}_{0}\right)$ satisfies $\left\|x\left(t ; t_{0}, \hat{x}_{0}\right)-x\left(t ; t_{0}, x_{0}\right)\right\|<\varepsilon$ on $\mathbb{I}$.

2. asymptotically stable if it is stable and there exists $\rho>0$ such that

a. the initial value problem (4) with initial condition $x\left(t_{0}\right)=\hat{x}_{0}$ is solvable on $\mathbb{I}$ for all $\hat{x}_{0} \in \mathbb{K}^{n}$ with $\left\|\hat{x}_{0}-x_{0}\right\|<\rho$

b. the solution $x\left(t ; t_{0}, \hat{x}_{0}\right)$ satisfies $\lim _{t \rightarrow \infty}\left\|x\left(t ; t_{0}, \hat{x}_{0}\right)-x\left(t ; t_{0}, x_{0}\right)\right\|=0$.

3. exponentially stable if it is stable and exponentially attractive, i.e., if there exist $\delta>0, L>0$, and $\gamma>0$ such that

a. the initial value problem (4) with initial condition $x\left(t_{0}\right)=\hat{x}_{0}$ is solvable on $\mathbb{I}$ for all $\hat{x}_{0} \in \mathbb{K}^{n}$ with $\left\|\hat{x}_{0}-x_{0}\right\|<\delta$;

b. the solution satisfies the estimate $\left\|x\left(t ; t_{0}, \hat{x}_{0}\right)-x\left(t ; t_{0}, x_{0}\right)\right\|<L e^{-\gamma\left(t-t_{0}\right)}$ on $\mathbb{I}$.

If $\delta$ does not depend on $t_{0}$, then we say the solution is uniformly (exponentially) stable. 
Note that one can transform the ODE (4) in such a way that a given solution $x\left(t ; t_{0}, x_{0}\right)$ is mapped to the trivial solution by simply shifting the arguments. When studying the stability of a selected solution, one may therefore assume without loss of generality that the selected solution is the trivial solution, and also that $t_{0}=0$.

One can immediately extend Definition 1 verbatim to DAEs. However, one has to be careful with the initial conditions and the inhomogeneities, since they are restricted due to the algebraic constraints in the system. This is, in particular, true if one considers the robustness of the stability concepts under perturbations to the system.

The following examples give an illustration for the possible difficulties in the robustness of the stability concepts for DAEs under small perturbations.

Example 1. Consider the homogeneous linear time-invariant DAE

$$
\left[\begin{array}{ll}
1 & 0 \\
0 & 0
\end{array}\right]\left[\begin{array}{c}
\dot{x}_{1} \\
\dot{x}_{2}
\end{array}\right]=\left[\begin{array}{ll}
0 & 1 \\
1 & 0
\end{array}\right]\left[\begin{array}{l}
x_{1} \\
x_{2}
\end{array}\right]
$$

which can be written as $\dot{x}_{1}=x_{2}, 0=x_{1}$ and has only the trivial solution $x_{1}=x_{2}=0$.

If we perturb (5) by a small $\varepsilon$ as

$$
\left[\begin{array}{ll}
1 & 0 \\
0 & 0
\end{array}\right]\left[\begin{array}{c}
\dot{x}_{1} \\
\dot{x}_{2}
\end{array}\right]=\left[\begin{array}{ll}
0 & 1 \\
1 & \varepsilon
\end{array}\right]\left[\begin{array}{l}
x_{1} \\
x_{2}
\end{array}\right]
$$

then solving the second equation of (6) for $x_{2}$ and substituting into the first equation, we obtain

$$
\dot{x}_{1}=-(1 / \varepsilon) x_{1} .
$$

Clearly, if $\varepsilon<0$, then the perturbed DAE (6) is unstable. If $\varepsilon>0$, then the system is asymptotically stable, but it qualitatively differs from the solution of the original system (5). For an arbitrarily prescribed initial value $x_{1}(0) \neq 0$, the initial value problem for (7) has a unique solution. Furthermore, the value of $x_{2}(0)$ is not required and is uniquely determined by $x_{1}(0)$. In fact, this small perturbation has changed the index of the DAE (5), see Definition 3 below.

If we add an inhomogeneity to these DAEs, then more essential differences appear.

In Example 1 the perturbation is affecting only the coefficient $A$. The situation is even more complicated if we allow perturbations in the coefficient of $\dot{x}$.

Example 2. Consider the well-known singularly perturbed system

$$
\left[\begin{array}{cc}
I_{n_{1}} & 0 \\
0 & \varepsilon I_{n_{2}}
\end{array}\right]\left[\begin{array}{c}
\dot{x}_{1} \\
\dot{x}_{2}
\end{array}\right]=\left[\begin{array}{ll}
A_{11} & A_{12} \\
A_{21} & A_{22}
\end{array}\right]\left[\begin{array}{l}
x_{1} \\
x_{2}
\end{array}\right]
$$

where $A_{i j}, i, j \in\{1,2\}$, are constant matrices of appropriate sizes, and $\varepsilon>0$ is a small parameter. Let us assume that $A_{22}$ is invertible. If $\varepsilon$ is set to 0 , then the leading matrix becomes singular, i.e., we have a DAE, and we can solve the second equation for $x_{2}$, and obtain the so-called underlying $O D E$

$$
\dot{x}_{1}=\left(A_{11}-A_{12} A_{22}^{-1} A_{21}\right) x_{1} .
$$

It is well known that for sufficiently small $\varepsilon$, the (asymptotic) stability of (8) depends not only on the stability of the so-called slow sub-system associated with the underlying ODE, but also on that of the so called fast sub-system $\dot{x}_{2}=A_{22} x_{2}$, see [26, 29, 55], associated with the algebraic equation.

In this example, the rank of the leading matrix is changed, when $\varepsilon$ moves from zero to a nonzero value. In the case $\varepsilon=0$, the initial condition must be consistent to ensure the existence of a solution, but obviously this is not required in the case of a nonzero $\varepsilon$. The difficulties increase if $A_{22}$ is singular and/or the leading matrix involves a singular perturbation of a more general structure.

The presented examples already indicate some of the possible difficulties which will be discussed in this paper. We present the analysis of robust exponential and asymptotic stability for linear DAEs with timeinvariant or time-varying coefficients. This is a relatively young topic, starting with the work in $[15,75]$, 
which generalized results on the distance to instability and the concept of stability radius for ODEs in [46] and [84].

The outline of the paper is as follows. In Section 2 we summarize recent results on the robust stability and stability radii for linear time-invariant DAEs. In Section 3 we study the robust stability of linear timevarying DAEs. Stability radii, their dependence on the data, and the robustness of stability spectra are analyzed. Some discussions and topics for future research close the paper.

\section{Robust stability of linear time-invariant DAEs}

In this section we study homogeneous linear time-invariant DAEs of the form

$$
E \dot{x}=A x, \quad t \in \mathbb{I},
$$

where $E$ and $A$ are given constant matrices in $\mathbb{K}^{n \times n}, \mathbb{K}=\mathbb{C}$ or $\mathbb{R}$.

Definition 2. A matrix pair $(E, A), E, A \in \mathbb{K}^{n \times n}$ is called regular if there exists $\lambda \in \mathbb{C}$ such that the determinant of $(\lambda E-A)$, denoted by $\operatorname{det}(\lambda E-A)$, is different from zero. Otherwise, if $\operatorname{det}(\lambda E-A)=0$ for all $\lambda \in \mathbb{C}$, then we say that $(E, A)$ is singular.

If $(E, A)$ is regular, then a complex number $\lambda$ is called a (generalized finite) eigenvalue of $(E, A)$ if $\operatorname{det}(\lambda E-A)=0$. The set of all (finite) eigenvalues of $(E, A)$ is called the (finite) spectrum of the pencil $(E, A)$ and denoted by $\sigma(E, A)$. If $E$ is singular and the pair is regular, then we say that $(E, A)$ has the eigenvalue $\infty$.

In the following we only consider regular pairs $(E, A)$. Such pairs can be transformed to WeierstraßKronecker canonical form, see [7,35,37], i.e., there exist nonsingular matrices $W, T \in \mathbb{C}^{n \times n}$ such that

$$
E=W\left[\begin{array}{cc}
I_{r} & 0 \\
0 & N
\end{array}\right] T^{-1}, A=W\left[\begin{array}{cc}
J & 0 \\
0 & I_{n-r}
\end{array}\right] T^{-1},
$$

where $I_{r}, I_{n-r}$ are identity matrices of indicated size, $J \in \mathbb{C}^{r \times r}$, and $N \in \mathbb{C}^{(n-r) \times(n-r)}$ are matrices in Jordan canonical form and $N$ is nilpotent. If $E$ is invertible, then $r=n$, i.e., the second diagonal block does not occur.

Definition 3. Consider a regular pair $(E, A)$ with $E, A \in \mathbb{K}^{n \times n}$ in Weierstraß-Kronecker form (10). If $r<n$ and $N$ has nilpotency index $v \in\{1,2, \ldots\}$, i.e., $N^{v}=0, N^{i} \neq 0$ for $i=1,2, \ldots, v-1$, then $v$ is called the index of the pair $(E, A)$ and the associated $D A E(9)$ and we write $\operatorname{ind}(E, A)=v$. If $r=n$ then the DAE has index $v=0$.

The finite spectrum $\sigma(E, A)$ is given by the spectrum of $\sigma(J)$ in the Weierstraß-Kronecker form (10) and it is easy to verify that for the degree of the characteristic polynomial $\operatorname{deg} \operatorname{det}(\lambda E-A)=\operatorname{rank} E=r$ holds if and only if ind $(E, A) \leq 1$.

For the DAE (9) with initial condition (2), there always exists a projection matrix $P \in \mathbb{K}^{n \times r}$ so that the projected initial condition $P\left(x\left(t_{0}\right)-x_{0}\right)=0$ is consistent, i.e., the DAE (9) with this initial condition has a unique solution, see [7,37]. Shifting again the solution to the trivial solution $x=0$, for a DAE of the form (9) with regular pair $(E, A), E, A \in \mathbb{K}^{n \times n}$ we say that this solution is exponentially stable if there exist $\delta>0, L>0$, and $\gamma>0$ such that the initial value problem

$$
E \dot{x}=A x, P\left(x(0)-\hat{x}_{0}\right)=0,
$$

is solvable on $\mathbb{I}$ for all $\hat{x}_{0} \in \mathbb{K}^{n}$ with $\left\|\hat{x}_{0}\right\|<\delta$, and the solution satisfies the estimate $\left\|x\left(t ; t_{0}, \hat{x}_{0}\right)\right\|<$ $L e^{-\gamma\left(t-t_{0}\right)}$ on II. If the trivial solution is exponentially stable, then we say that (9) is exponentially stable.

We remark that for linear time-invariant systems, the concept of exponential stability is equivalent to that of asymptotic stability and hence we do not have to distinguish these concepts, and discuss only asymptotic stability as is usually done in the literature.

Using the canonical form (10), one can easily verify the following statement, see e.g., [15]. 
Proposition 1. Consider a DAE of the form (9) with regular pair (E,A), E,A $\in \mathbb{K}^{n \times n}$. System (9) is asymptotically stable if and only if the pair $(E, A)$ is asymptotically stable, i.e., the finite spectrum satisfies $\sigma(E, A) \subset \mathbb{C}^{-}$, where $\mathbb{C}^{-}$denotes the open left-half complex plane.

After introducing the basic notation, in the next subsection we discuss the stability radius of a DAE.

\subsection{Stability radii for lime time-invariant DAES}

In this section we study the behavior of the finite spectrum of a regular pair $(E, A)$ under structured perturbations in the matrices $E, A$. Suppose that the system (9) is asymptotically stable and consider a perturbed system

$$
\left(E+B_{1} \Delta_{1} C_{1}\right) \dot{x}=\left(A+B_{2} \Delta_{2} C_{2}\right) x,
$$

where $\Delta_{i} \in \mathbb{K}^{m_{i} \times q_{i}}(i=1,2)$ are perturbations and $B_{i} \in \mathbb{K}^{n \times m_{i}}, C_{i} \in \mathbb{K}^{q_{i} \times n}$ are given matrix pairs that restrict the structure of the perturbations. The matrix pair $\left(B_{1} \Delta_{1} C_{1}, B_{2} \Delta_{2} C_{2}\right)$ is called a structured perturbation. For simplicity, let us consider the case that the restricting matrices satisfy $C_{1}=C_{2}=C$ (alternatively we could consider the other simplifying case that $B_{1}=B_{2}$ ). Set

$$
\Delta=\left[\begin{array}{l}
\Delta_{1} \\
\Delta_{2}
\end{array}\right], B=\left[\begin{array}{ll}
B_{1} & B_{2}
\end{array}\right]
$$

and introduce $m=m_{1}+m_{2}$ and $q=q_{1}=q_{2}$. Then we consider the set of destabilizing perturbations

$$
\mathscr{V}_{\mathbb{K}}(E, A ; B, C)=\left\{\Delta \in \mathbb{K}^{m \times q},(11) \text { is singular or not asymptotically stable }\right\}
$$

and have the following definition.

Definition 4. The structured stability radius of (9) subject to structured perturbations as in (11) is defined by

$$
r_{\mathbb{K}}(E, A ; B, C)=\inf \left\{\|\Delta\|, \Delta \in \mathscr{V}_{\mathbb{K}}(E, A ; B, C)\right\},
$$

where $\|$.$\| is a matrix norm induced by a vector norm. Depending on \mathbb{K}=\mathbb{C}$ or $\mathbb{K}=\mathbb{R}$, we talk about the complex or the real stability radius, respectively.

Obviously, we have the estimate

$$
r_{\mathbb{C}}(E, A ; B, C) \leq r_{\mathbb{R}}(E, A ; B, C) .
$$

To obtain a computable formula for the complex stability radius, one introduces the matrix functions

$$
G_{1}(s)=-s C(s E-A)^{-1} B_{1}, G_{2}(S)=C(s E-A)^{-1} B_{2}, G(s)=\left[G_{1}(s) G_{2}(s)\right],
$$

for $s \in \mathbb{C}$. Denoting by $i \mathbb{R}$ the imaginary axis of the complex plane, the following result is analogous to that for linear time-invariant ODEs of [47].

Theorem 1. Suppose that the matrix pencil $(E, A)$ is regular and asymptotically stable. Then with respect to any matrix norm induced by a vector norm, the complex stability radius of (9) has the representation

$$
r_{\mathbb{C}}(E, A ; B, C)=\left\{\sup _{s \in i \mathbb{R}}\|G(s)\|\right\}^{-1} .
$$

Proof. The proof can be obtained by using the same techniques as in [27, 28, 32].

Note that in $[27,28]$ the complex structured stability radius is considered with respect to perturbations either in $E$ or in $A$, i.e., either $B_{1}=0$ or $B_{2}=0$. In [32], formula (12) has been proven for perturbations in both $E, A$. Note further that these papers discuss both the continuous-time and the discrete-time case. Furthermore, it has been shown in [32] that it is always possible to construct a rank 1 destabilizing perturbation $\Delta$, with a norm that approximates the value of $r_{\mathbb{C}}$ within an arbitrarily prescribed accuracy. 
Remark 1 . The concept of stability radius can be extended to more general sets. Suppose that all the eigenvalues of the unperturbed matrix pencil lie in a prescribed open subset $\mathbb{C}_{g}$ of the complex plane. Then we want to determine the largest perturbations that the system can tolerate so that its spectrum remains in $\mathbb{C}_{g}$. In the asymptotic stability analysis of differential equations, the open subset $\mathbb{C}_{g}$ is chosen to be $\mathbb{C}^{-}$. As in the other cases, it is trivial to obtain a formula of a $\mathbb{C}_{g}$-stability radius analogously to (12). In fact, we simply replace $i \mathbb{R}$ by the boundary set of $\mathbb{C}_{b}=\mathbb{C} \backslash \mathbb{C}_{g}$. As a consequence of the definition, the strict positivity of a $\mathbb{C}_{g}$-stability radius with a relevant subset $\mathbb{C}_{g}$ implies the continuity of the spectrum with respect to the data.

Unlike for the complex stability radius, a general formula for the real stability radius measured by an arbitrary matrix norm is not available. However, if we consider as vector norm the Euclidean norm, then a computable formula has been obtained in [74]. This formula is based on the notion of real/complex structured singular values, which for a given $M \in \mathbb{K}^{p \times m}$, are defined by

$$
\mu_{\mathbb{K}}(M)=\left[\inf \left\{\sigma_{1}(\Delta), \Delta \in \mathbb{K}^{m \times p}, \text { and } \operatorname{det}(I-\Delta M)=0\right\}\right]^{-1},
$$

respectively, depending on $\mathbb{K}=\mathbb{C}$ or $\mathbb{K}=\mathbb{R}$. Here $\sigma_{1}(\Delta)$ denotes the largest singular value, see [36], of the matrix $\Delta$.

Clearly, if $M$ is real, then the complex and the real structured singular values coincide. While for the complex structured singular values, the formula $\mu_{\mathbb{C}}(M)=\sigma_{1}(M)$ follows trivially, for the real case the formula is more sophisticated.

Proposition 2. [74] The real structured singular value of $M \in \mathbb{K}^{m \times q}$ is given by

$$
\mu_{\mathbb{R}}(M)=\inf _{\gamma \in(0,1]} \sigma_{2}\left[\begin{array}{cc}
\operatorname{Re} M & -\gamma \operatorname{Im} M \\
\frac{1}{\gamma} \operatorname{Im} M & \operatorname{Re} M
\end{array}\right],
$$

where $\sigma_{2}(A)$ denotes the second largest singular value of $A$.

Using the definition of the stability radius with respect to the Euclidean norm, we thus have that

$$
\begin{aligned}
r_{\mathbb{K}}(E, A ; B, C) & =\inf \left\{\sigma_{1}(\Delta), \Delta \in \mathscr{V}_{\mathbb{K}}(E, A ; B, C)\right\} \\
& =\inf _{s \in i \mathbb{R}} \inf \left\{\sigma_{1}(\Delta), \Delta \in \mathbb{K}^{m \times q} \text { and } \operatorname{det}\left(s\left(E+B_{1} \Delta_{1} C\right)-\left(A+B_{2} \Delta_{2} C\right)\right)=0\right\} \\
& =\inf _{s \in i \mathbb{R}} \inf \left\{\sigma_{1}(\Delta), \Delta \in \mathbb{K}^{m \times q} \text { and } \operatorname{det}\left(I+(s E-A)^{-1}\left(s B_{1} \Delta_{1} C-B_{2} \Delta_{2} C\right)\right)=0\right\} \\
& =\inf _{s \in \mathbb{R}} \inf \left\{\sigma_{1}(\Delta), \Delta \in \mathbb{K}^{m \times q} \text { and } \operatorname{det}(I-\Delta G(s))=0\right\} \\
& =\left\{\sup _{s \in \mathbb{R} \mathbb{R}} \mu_{\mathbb{K}}(G(s))\right\}^{-1},
\end{aligned}
$$

and hence we obtain the following theorem.

Theorem 2. [74] Suppose that the matrix pencil $(E, A)$ is regular and asymptotically stable. Then the structured stability radii of (9), measured in Euclidean norm, are given by

$$
r_{\mathbb{C}}(E, A ; B, C)=\left\{\sup _{s \in i \mathbb{R}} \sigma_{1}(G(s))\right\}^{-1},
$$

and

$$
r_{\mathbb{R}}(E, A ; B, C)=\left\{\sup _{s \in i \mathbb{R}} \inf _{\gamma \in(0,1]} \sigma_{2}\left[\begin{array}{cc}
\operatorname{Re} G(s) & -\gamma \operatorname{Im} G(s) \\
\frac{1}{\gamma} \operatorname{Im} G(s) & \operatorname{Re} G(s)
\end{array}\right]\right\}^{-1}
$$

respectively.

It is important to note that the presented results on the structured stability radii do not reflect the fact that an eigenvalue at $\infty$ may become finite or conversely a finite eigenvalue may move to $\infty$, i.e., it may happen that the index of the pair $(E, A)$ changes or that the pair becomes singular.

An increase of the index is not problematic for homogeneous systems with zero initial conditions, since the part of the solution associated with the infinite spectrum is always 0 . In the case of inhomogeneous systems or nonzero initial conditions, however, an increase of the index may lead to a loss of solvability 
of the equation due to inconsistent initial values or a lack of smoothness for the inhomogeneity. As we have demonstrated in Examples 1 and 2, this can even happen with infinitesimally small perturbations. Furthermore, while the stability radii of an ODE are always strictly positive, those of a DAE may be zero. To see this, considering a pair in canonical form (10), we have

$$
(s E-A)^{-1}=T\left[\begin{array}{cc}
\left(s I_{r}-J\right)^{-1} & 0 \\
0 & -\sum_{i=0}^{k-1}(s N)^{i}
\end{array}\right] W^{-1} .
$$

It then obvious that if $N \neq 0$, then $\left\|G_{1}(s)\right\|$ and $\left\|G_{2}(s)\right\|$ may tend to $\infty$ as $|s| \rightarrow \infty$, which implies that $r_{\mathbb{C}}=0$. Hence, the perturbations in (11) must be further restricted so that the stability radii are strictly positive.

Partitioning the restriction matrices $C, B$ (after transformation to Weierstraß-Kronecker form) as

$$
C T=\left[C_{1} C_{2}\right], \quad W^{-1} B_{1}=\left[\begin{array}{l}
B_{11} \\
B_{12}
\end{array}\right], \quad W^{-1} B_{2}=\left[\begin{array}{l}
B_{21} \\
B_{22}
\end{array}\right],
$$

according to the structure of (10), it is easy to see that if $\operatorname{ind}(E, A)=1$ then

$$
\sup _{s \in i \mathbb{R}}\left\|G_{1}(s)\right\|<\infty \text { if and only if } C_{2} B_{12}=0
$$

and if $\operatorname{ind}(E, A)>1$ then

$$
\sup _{s \in i \mathbb{R}}\left\|G_{2}(s)\right\|<\infty \text { if and only if } C_{2} B_{12}=0 \text { and } C_{2} B_{22}=0 .
$$

These observations are summarized in the following result.

Proposition 3. Consider a regular pair $(E, A)$ and the associated $D A E$ of the form (9). If $\operatorname{ind}(E, A)=1$, then the structured stability radii of (9) are strictly positive if and only if $C_{2} B_{12}=0$. If $\operatorname{ind}(E, A)>1$, then the structured stability radii of (9) are strictly positive if and only if $C_{2} B_{12}=0$ and $C_{2} B_{22}=0$, where the transformed structure matrices are defined by (15).

Moreover, if $C_{2}$ is of full rank, then $r_{\mathbb{K}}(E, A, B, C)>0$ if and only if $B_{12}=0$ for the case $\operatorname{ind}(E, A)=1$ and $B_{12}=B_{22}=0$ for the case ind $(E, A)>1$.

It follows that the perturbations have to be further restricted by choosing

$$
B_{1}=W\left[\begin{array}{c}
B_{11} \\
0
\end{array}\right]
$$

if $\operatorname{ind}(E, A)=1$ and

$$
B_{1}=W\left[\begin{array}{c}
B_{11} \\
0
\end{array}\right], \quad B_{2}=W\left[\begin{array}{c}
B_{21} \\
0
\end{array}\right]
$$

if $\operatorname{ind}(E, A)>1$.

Definition 5. [15] A structured perturbation as in (11) is called admissible if it does not alter the nilpotency structure of the Weierstrass-Kronecker form (10) of $(E, A)$, i.e., the nilpotent matrix $N$ and the corresponding left invariant subspace associated with the eigenvalue $\infty$ are preserved.

In the case that ind $(E, A)=1$, one has the following characterization of admissible perturbations.

Proposition 4. [15] Consider the regular DAE (9) with $\operatorname{ind}(E, A)=1$, subject to a general unstructured perturbation

$$
(E+F) \dot{x}=(A+H) x .
$$

Then there exist an orthogonal matrix $P$ and a permutation matrix $Q$ such that

$$
P E Q=\left[\begin{array}{cc}
E_{11} & E_{12} \\
0 & 0
\end{array}\right], \quad P A Q=\left[\begin{array}{ll}
A_{11} & A_{12} \\
A_{21} & A_{22}
\end{array}\right],
$$


where $\operatorname{rank}\left[E_{11}, E_{12}\right]=\operatorname{rank} E=r$, and $\operatorname{rank} A_{22}=n-r$. Furthermore, if $(F, H)$ is an admissible perturbation, then

$$
P F Q=\left[\begin{array}{cc}
F_{11} & F_{12} \\
0 & 0
\end{array}\right], \quad P H Q=\left[\begin{array}{ll}
H_{11} & H_{12} \\
H_{21} & H_{22}
\end{array}\right] .
$$

Note that in Proposition 4, the transformation by the matrices $P, Q$ does not change the structure, the stability, and consequently, the stability radii of (9). Note further that Proposition 3 can also be used to characterize admissible perturbations for the case ind $(E, A)>1$.

After these observations we can introduce the distance to the nearest pair with a different nilpotency structure

$$
d_{\mathbb{K}}(E, A ; B, C)=\inf \left\{\|\Delta\|, \Delta \in \mathbb{K}^{m \times q} \text { and (11) does not preserve the nilpotency structure }\right\},
$$

and obtain the following result, see [5].

Theorem 3. Consider a regular DAE with Weierstraß-Kronecker form (10), subject to transformed perturbations satisfying (16) and (17). Then the distance to the nearest system with a different nilpotency structure is given by

$$
d_{\mathbb{K}}(E, A ; B, C)=\left\|\left[C_{1} B_{11} C_{2} B_{22}\right]\right\|^{-1}
$$

if $\operatorname{ind}(E, A)=1$ and

$$
d_{\mathbb{K}}(E, A ; B, C)=\left\|C_{1} B_{11}\right\|^{-1},
$$

if $\operatorname{ind}(E, A)>1$. Moreover, $d_{\mathbb{C}}(E, A ; B, C)=d_{\mathbb{R}}(E, A ; B, C)$.

Proof. The proof is similar to that for the stability radii, see Remark 1. The nilpotency structure of the perturbed system (11) is preserved if and only if the perturbed matrix

$$
\left[\begin{array}{cc}
I+B_{11} \Delta_{1} C_{1} & B_{11} \Delta_{1} C_{2} \\
B_{22} \Delta_{2} C_{2} & I+B_{22} \Delta_{2} C_{2}
\end{array}\right]
$$

is nonsingular in the case $\operatorname{ind}(E, A)=1$ and if $I+B_{11} \Delta_{1} C_{1}$ is nonsingular in the case $\operatorname{ind}(E, A)>1$. Thus, in both cases we have a problem of characterizing the distance of a matrix to singularity, which we obtain by taking $\mathbb{C}_{g}=\mathbb{C} \backslash\{0\}$. For the equality of the complex and the real stability radii, we note that if the data are real, then the smallest perturbation that makes a matrix singular can always be chosen to be real [36]. (

We summarize the results in the following theorem.

Theorem 4. Consider a regular and asymptotically stable DAE (9) with Weierstraß-Kronecker form (10). Let the perturbation structure satisfy (16) and (17) for index $\operatorname{ind}(E, A)=1$ and $\operatorname{ind}(E, A)>1$, respectively. If $\|\Delta\|<r_{\mathbb{K}}(E, A, B, C)$, then the perturbed $D A E(11)$ preserves not only the regularity and the asymptotic stability, but also the nilpotency structure of the DAE (9). In other words, we obtain the structured stability radii as

$$
r_{\mathbb{K}}(E, A ; B, C)=\inf \{\|\Delta\|, \Delta \in \mathscr{V}(E, A ; B, C) \text { or (11) has different nilpotency structure }\} .
$$

These structured stability radii are given by the formulas (12), (13), and (14), respectively.

Proof. Assuming (16) and (17), respectively, it is easy to check that

$$
\lim _{s \rightarrow \infty} G_{1}(s)=C_{1} B_{11}, \text { and } \lim _{s \rightarrow \infty} G_{2}(s)=C_{2} B_{22} .
$$

Since $\sup _{s \in i \mathbb{R}}\|G(s)\| \geq\|G(\infty)\|$, the statement for the case $\mathbb{K}=\mathbb{C}$ follows directly from Theorem 1 and Theorem 3.

If all the data are real, then $G(\infty)$ is real as well. Since the complex and the real structured singular values of $G(\infty)$ are equal, again by Theorem 3 , the same statement holds for the case $\mathbb{K}=\mathbb{R}$. $\square$ 
Remark 2. The first results for stability radii for linear time-invariant DAEs of index one were given in [75] and [15]. In [75] only unstructured perturbations in $A$ were considered and the formula for the unstructured complex stability radius measured in Euclidean norm is exactly a special case of (13) with restriction matrices $B_{1}=0$ and $B_{2}=C=I$. A more general result was obtained in [15], where the authors considered admissible perturbations as in Proposition 4 and formulated the complex stability radius using the Frobenius norm which is not a matrix norm induced by a vector norm. However, using the fact that the Frobenius norm gives an upper bound for the Euclidean norm, the stability radii in Frobenius norm are upper bounds for those in Euclidean norm. On the other hand, in the proof of Theorem 1, a rank one destabilizing perturbation can be constructed whose (Euclidean) norm approximates the true value of the stability radius with an arbitrarily small accuracy. Since the Euclidean and the Frobenius norm of a rank one matrix are equal, the formula of the complex stability radius given in [15] and (13) yield the same value, i.e., the former one can be considered as a special case of (12) and (13).

Remark 3. In many applications, one does not only have static perturbations as in (11), but also linear timevarying, nonlinear or even nonlinear dynamic perturbations. In the context of regular DAEs (9) of index at most one, if perturbations are admitted in $A$ only, then it is possible to extend the concept of structured stability radii with respect to linear time-varying, nonlinear, or nonlinear dynamic perturbations. It can be shown that all the complex structured stability radii with respect to different classes of perturbations are equal as it is well known in the ODE case [49], see also Corollary 2 below.

Remark 4. Numerical algorithms for computing the stability radii for ODEs are proposed in a number of works, e.g., see [4, 6, 12, 34, 38, 39, 40, 42, 45, 71, 72, 73, 81]. Some extensions to DAEs are discussed in [5]. Since the robust stability of a linear time-invariant system is closely related to the sensitivity of the spectrum, the characterization and the computation of stability radii is also very closely related to the topics of spectral value sets $[44,50]$ and pseudospectra $[11,83]$.

Remark 5. Robustness questions can also be discussed for other fundamental concepts of control theory such as controllability, observability, stabilizability, or detectability. These concepts have been extended to DAEs in many different publications, see e.g., $[8,9,10,17,21,68,70]$. It is natural to analyze the robustness of these properties when the control systems are subject to uncertain perturbations, which leads to similar distance problems as for robust stability. For ODEs, such distance problems are extensively investigated in a number of works, e. g., see $[52,71]$ and the references therein. Results on the controllability radius for linear time-invariant descriptor systems are given in $[13,58,59,80]$.

It is well known that solutions of DAEs are more sensitive to data than those of ODEs. This topic has been discussed in [67] for a perturbed index two DAE in semi-explicit form and in $[24,82]$ for general singularly perturbation problems of DAEs. But a general perturbation theory for linear time-invariant DAEs is still open. This is partly due to the fact that no complete characterization of the distance to the nearest singular pencil is available [14].

\subsection{Dependence of stability radii on the data}

In view of the numerical computation of the stability radii, a natural question is whether the structured stability radii $r_{\mathbb{K}}(E, A ; B, C)$ depend continuously on the data $E, A, B, C$. In the ODE case, i.e., $E=I$ and if only $A$ is perturbed, it was shown in [48] that the complex structured radius depends continuously on data, but the real one does not. Extending these results to DAEs, it follows that the complex structured stability radius $r_{\mathbb{C}}(E, A ; B, C)$ depends continuously on the data, provided that the nilpotency structure is preserved, i.e., we are restricted to the set of DAEs (9) that have the same nilpotency structure and to the set of admissible perturbations. In $[29,30]$ the robust stability of the parameterized DAE system

$$
(E+\varepsilon F) \dot{x}=A x,
$$

is considered, where $\varepsilon>0$ is a small parameter and the unperturbed DAE $(\varepsilon=0)$ is assumed to be regular, of index at most one and asymptotically stable. The classical singularly perturbed system (8) is a special 
case of this more general system. If $\varepsilon F$ belongs to the class of admissible perturbations characterized by Proposition 4, then it is easily shown that the complex structured stability radius depends continuously on the parameter $\varepsilon$. This, however, is not the case when the appearance of $\varepsilon F$ changes the index and/or the number of finite eigenvalues, i.e., the nilpotency structure of $(E, A)$.

Sufficient conditions can be given to ensure that (18) is asymptotically stable for all sufficiently small and positive $\varepsilon$. Namely, if the unperturbed DAE $(\varepsilon=0)$ and the fast subsystem (that is associated with the algebraic part of the DAE) are simultaneously asymptotically stable, then for all sufficiently small $\varepsilon$, so is the parameterized system (18). Furthermore, the complex structured stability radius of (18) converges to the minimum of the stability radius of the reduced system and that of the fast subsystem. This implies that the stability radius of (18) may be discontinuous at $\varepsilon=0$, when the nilpotency structure is no longer invariant. As a special case, the result for the robust stability of (8), investigated in [25] by a different approach, then follows immediately.

Remark 6. In [61], the robust stability of a DAE subject to perturbations of the form $E \dot{x}=(A+\varepsilon H) x$ is considered, where $E, A$ are given as in (9), $H$ is a given matrix, and $\varepsilon$ is a uncertain parameter. Assuming that the unperturbed system is regular, of index at most one and asymptotically stable, a computable formula for the maximal stability interval $\left(\varepsilon_{1}, \varepsilon_{2}\right)$ is derived, i.e., the perturbed system retains the index and is asymptotically stable for all $\varepsilon \in\left(\varepsilon_{1}, \varepsilon_{2}\right)$.

A somewhat more general and extensive analysis of stability radii for DAEs is given in [5], considering both the structure and the spectrum, which are treated separately based on the notion of structured singular values. This approach makes the characterization of stability radii for higher index DAEs possible as well as that for the special uncertainty structure of affine perturbations. The latter one also extends the result in [61] to the case when both coefficient matrices are perturbed with a one-parameter family. In addition, the work in [5] is partially devoted to robust stability of second order DAEs with applications in electrical networks.

In [28], complex structured stability radii for the discrete-time analogue of DAEs, i.e., singular difference equations are analyzed. In particular, the complex structured stability radius of the discretized system of (9) using the implicit Euler method is shown to converge to the corresponding one of the continuous-time system as the stepsize tends to zero.

It has been shown in [79] that for the class of positive systems the complex and the real stability radii coincide and they are easily computable. Attempts to extend this result to DAEs and to other implicit dynamic equations are presented in [27] and [32], respectively.

\section{Robust stability of linear time-varying DAEs}

In this section, we investigate the exponential and asymptotic stability and its robustness for linear timevarying DAEs of the form

$$
E \dot{x}=A x, t \in \mathbb{I},
$$

with matrix functions $E, A \in C\left(\mathbb{I}, \mathbb{K}^{n \times n}\right), \mathbb{K} \in\{\mathbb{C}, \mathbb{R}\}$.

Analyzing the different stability concepts for (19) is, however, much more complicated than for linear time-invariant systems. Even if for all $t \in \mathbb{I}$, the finite eigenvalues of $(E(t), A(t))$ have negative real part, system (19) may be unstable, as many well-known examples demonstrate for the ODE case, see e.g., [51].

Example 3. For all $t \in \mathbb{R}$

$$
A(t)=\left[\begin{array}{cc}
\cos ^{2}(3 t)-5 \sin ^{2}(3 t) & -6 \cos (3 t) \sin (3 t)+3 \\
-6 \cos (3 t) \sin (3 t)+3 & \sin ^{2}(3 t)-5 \cos ^{2}(3 t)
\end{array}\right]
$$

has a double eigenvalue at -2 but the solution of $\dot{x}=A x$, with $x(0)=\left[\begin{array}{c}c_{1} \\ 0\end{array}\right]$ is given by $x(t)=$ $\left[\begin{array}{c}c_{1} e^{t} \cos (3 t) \\ -c_{1} e^{t} \sin (3 t)\end{array}\right]$, which is obviously unstable. 
In the following we assume that (19) is of index at most one, in the sense of the tractability index [37] or that it is strangeness-free in the sense of the strangeness-index [56]. Let us briefly introduce these index concepts.

We use the following standard notation as in [31, 54]. Let $X, Y$ be finite dimensional vector spaces. For every $p, 1 \leq p<\infty$ and $s, t, t_{0} \leq s<t<\infty$, we denote by $L_{p}(s, t ; X)$ the space of measurable functions $f$ with values in $X$ and norm $\|f\|_{p}:=\left(\int_{s}^{t}\|f(\rho)\|^{p} d \rho\right)^{1 / p}<\infty$ and by $L_{\infty}(s, t ; X)$ the space of measurable and essentially bounded functions $f$ with $\|f\|_{\infty}:=\operatorname{ess} \sup _{\rho \in[s, t]}\|f(\rho)\|$. We also consider the spaces $L_{p}^{l o c}\left(t_{0}, \infty ; X\right)$ and $L_{\infty}^{l o c}\left(t_{0}, \infty ; X\right)$, which contain all functions $f \in L_{p}(s, t ; X)$ and $f \in L_{\infty}(s, t ; X)$ for some $s, t, t_{0} \leq s<t<\infty$, respectively. We, furthermore, use the notation $\mathscr{L}\left(L_{p}\left(t_{0}, \infty ; X\right), L_{p}\left(t_{0}, \infty ; Y\right)\right)$ to denote the Banach space of linear bounded operators $\mathbb{P}$ from $L_{p}\left(t_{0}, \infty ; X\right)$ to $L_{p}\left(t_{0}, \infty ; Y\right)$ supplied with the norm

$$
\|\mathbb{P}\|:=\sup _{x \in L_{p}\left(t_{0}, \infty ; X\right),\|x\|=1}\|\mathbb{P} x\|_{L_{p}\left(t_{0}, \infty ; Y\right)} .
$$

To introduce the tractability index and the projector chain approach, see e.g., [37, 60], if $E, A \in$ $L_{\infty}^{l o c}\left(0, \infty ; \mathbb{K}^{n \times n}\right)$, then for $S:=\operatorname{ker} E$ there exists an absolutely continuous projector $Q$ onto $S$, i.e., $Q \in C\left(0, \infty ; \mathbb{K}^{n \times n}\right), Q$ is differentiable almost everywhere, $Q^{2}=Q$, and $\operatorname{Im} Q=S$ for all $t \in \mathbb{I}$. If we assume in addition that $\dot{Q} \in L_{\infty}^{l o c}\left(0, \infty ; \mathbb{K}^{n \times n}\right)$, then $P=I-Q$ is a projector along $S$ and system (19) can be rewritten in the form

$$
E \frac{d}{d t}(P x)=\widehat{A} x
$$

where $\widehat{A}:=A+E \dot{P} \in L_{\infty}^{l o c}\left(0, \infty ; \mathbb{K}^{n \times n}\right)$. Setting $G:=E-\widehat{A} Q$, the DAE (2.1) is said to be tractable of index one, if $G(t)$ is invertible for almost every $t \in[0, \infty)$ and $G^{-1} \in L_{\infty}^{l o c}\left(0, \infty ; \mathbb{K}^{n \times n}\right)$.

Multiplying both sides of (20) by $P G^{-1}, Q G^{-1}$, we obtain

$$
\begin{aligned}
& \frac{d}{d t}(P x)=\left(\frac{d}{d t} P+P G^{-1} \widehat{A}\right) P x, \\
& Q x=Q G^{-1} \widehat{A} P x,
\end{aligned}
$$

which decomposes the DAE into a differential part and an algebraic part. With $z=P x$, the dynamics of the system is given by the inherent $O D E$

$$
\dot{z}=\left(\dot{P}+P G^{-1} \widehat{A}\right) z
$$

of (19). Let $\Phi_{0}(t, s)$ denote the fundamental solution associated with the inherent ODE (21), i.e., the matrix function satisfying

$$
\frac{d}{d t} \Phi_{0}(t, s)=\left(\dot{P}+P G^{-1} \widehat{A}\right) \Phi_{0}(t, s), \Phi_{0}(s, s)=I,
$$

for $t>s \geq 0$, then the fundamental solution operator generated by (19) is defined by

$$
E \frac{d}{d t} \Phi(t, s)=A \Phi(t, s), P(s)(\Phi(s, s)-I)=0
$$

and $\Phi$ can be expressed as $\Phi(t, s)=\left(I+Q G^{-1} \widehat{A}\right)(t) \Phi_{0}(t, s) P(s)$.

The concept of the strangeness index uses the DAE and its derivatives to construct a so-called strangeness-free DAE with the same solution [56]. In the homogenous case this strangeness-free system has the form

$$
E \dot{x}=A x, \quad t \in \mathbb{I},
$$

where $E=\left[\begin{array}{c}E_{1} \\ 0\end{array}\right], A=\left[\begin{array}{l}A_{1} \\ A_{2}\end{array}\right]$, with $E_{1} \in C\left(\mathbb{I}, \mathbb{K}^{d \times n}\right)$ and $A_{2} \in C\left(\mathbb{I}, \mathbb{K}^{(n-d) \times n}\right)$ such that the matrix $\hat{E}(t):=$ $\left[\begin{array}{l}E_{1}(t) \\ A_{2}(t)\end{array}\right]$ is invertible for all $t \in \mathbb{I}$.

By using a global kinematic equivalence transformation, see [62, Remark 13], (22) can be transformed to the special form,

$$
\left[\begin{array}{cc}
\tilde{E}_{11} & \tilde{E}_{12} \\
0 & 0
\end{array}\right] \dot{\tilde{x}}=\left[\begin{array}{cc}
\tilde{A}_{11} & \tilde{A}_{12} \\
0 & \tilde{A}_{22}
\end{array}\right] \tilde{x}, \quad t \in \mathbb{I} \text {. }
$$


so that the underlying ODE is given by $E_{11} \dot{x}_{1}=A_{11} x_{1}$ with nonsingular $E_{11}$.

A matrix function $\Phi \in C^{1}\left(\mathbb{I}, \mathbb{R}^{n \times d}\right)$ is called minimal fundamental solution matrix of the strangenessfree DAE (22) if each of its columns is a solution to (22) and $\operatorname{rank} \Phi(t)=d$, for all $t \in \mathbb{I}$.

In the following we assume that the DAE is of index at most one or alternatively strangeness-free. These conditions are equivalent if the coefficients are sufficiently smooth [69].

The characterization of the different stability concepts for linear variable coefficient ODEs is well established via the concepts of Bohl and Lyapunov exponents, [3, 22] and Sacker-Sell spectra [23, 78]. These concepts were extended from ODEs to DAEs in $[18,62,63]$. In the following, for simplicity, we assume that (19) possesses piecewise continuous coefficients.

To analyze exponential stability, we introduce first the Bohl exponent.

Definition 6. The Bohl exponent for an index one system of the form (19) with fundamental solution $\Phi$ is given by

$$
\begin{aligned}
k_{B}(E, A)= & \inf \left\{-\alpha \in \mathbb{R} ; \text { there exists } L_{\alpha}>0\right. \\
& \text { such that for all } \left.t \geq t_{0} \geq 0:\left\|\Phi\left(t, t_{0}\right)\right\| \leq L_{\alpha} e^{-\alpha\left(t-t_{0}\right)}\right\} .
\end{aligned}
$$

It follows that (19) is exponentially stable if and only if its largest Bohl exponent is negative.

Analogously to the ODE case (see [22]), using the fundamental solution operator $\Phi$, it follows that the Bohl exponent of (19) is finite if and only if $\sup _{0 \leq|t-s| \leq 1}\|\Phi(t, s)\|<\infty$. Furthermore, if the Bohl exponent of (19) is finite, then it can be determined by

$$
k_{B}(E, A)=\limsup _{s, t-s \rightarrow \infty} \frac{\ln \|\Phi(t, s)\|}{t-s} .
$$

In [18], various properties of the Bohl exponent, as well as the connection between the exponential stability of (19) and the boundedness of solutions to nonhomogeneous DAE with bounded inhomogeneity are investigated.

For ODEs, the asymptotic stability of solutions can be characterized by the Lyapunov exponents, see [66]. The extension of the theory of Lyapunov exponents to linear time-varying DAEs has been given in $[19,20,62,63,64]$, using either the projector-based tractability index or the derivative array-based strangeness index approach.

Definition 7. For a given minimal fundamental solution matrix $\Phi$ of a strangeness-free DAE system of the form (22), and for $1 \leq i \leq d$, we introduce

$$
\lambda_{i}^{u}=\limsup _{t \rightarrow \infty} \frac{1}{t} \ln \left\|\Phi(t) e_{i}\right\| \text { and } \lambda_{i}^{\ell}=\liminf _{t \rightarrow \infty} \frac{1}{t} \ln \left\|\Phi(t) e_{i}\right\|,
$$

where $e_{i}$ denotes the $i$-th unit vector and $\|\cdot\|$ denotes the Euclidean norm. The columns of a minimal fundamental solution matrix form a normal basis if $\sum_{i=1}^{d} \lambda_{i}^{u}$ is minimal. The $\lambda_{i}^{u}, i=1,2, \ldots, d$ belonging to a normal basis are called (upper) Lyapunov exponents and the intervals $\left[\lambda_{i}^{\ell}, \lambda_{i}^{\mu}\right], i=1,2, \ldots, d$, are called Lyapunov spectral intervals.

The strangeness-free DAE system (22) then is asymptotically stable if and only if the largest upper Lyapunov exponent is negative. Note that for linear time-invariant DAEs (9), the Lyapunov exponents are exactly the real parts of the finite eigenvalues of pencil $(E, A)$.

This brings us to another major difference between linear time-invariant and linear time-varying DAEs. The following example shows is that an infinitesimally small time-varying perturbation applied to both coefficient matrices may change the asymptotic stability, even if the perturbation does not change the index.

Example 4. [63] Consider the system $\dot{x}_{1}=-x_{1}, 0=x_{2}$, which is strangeness-free and asymptotically stable. For the perturbed DAE

$$
\left(1+\varepsilon^{2} \sin (2 n t)\right) \dot{x}_{1}-\varepsilon \cos (n t) \dot{x}_{2}=-x_{1}, 0=-2 \varepsilon \sin (n t) x_{1}+x_{2},
$$


where $\varepsilon$ is a small parameter and $n$ is a given number, from the second equation of (24), we obtain $x_{2}=$ $2 \varepsilon \sin n t x_{1}$. Differentiating this expression for $x_{2}$ and inserting the result into the first equation, after some elementary calculations, we obtain $\dot{x}_{1}=\left(-1+n \varepsilon^{2}+n \varepsilon^{2} \cos (2 n t)\right) x_{1}$. Explicit integration yields $x_{1}(t)=$ $e^{\left(-1+n \varepsilon^{2}\right) t+\varepsilon^{2} \sin (2 n t) / 2} x_{1}(0)$. Clearly, even if $\varepsilon$ is arbitrarily small (hence the perturbation in the coefficient matrices is arbitrarily small in the sup-norm), (24) may become unstable if $n$ is sufficiently large.

\subsection{Stability radii for linear time-varying DAEs}

In this section we discuss the stability radii for linear DAEs with variable coefficients. Formulas for the stability radii of exponential stability were derived in $[18,31]$ extending the results for ODEs in [43, 54].

We assume that the DAE is of index at most one and discuss perturbed systems

$$
E \dot{x}=(A+H) x, \quad t \in \mathbb{I},
$$

with a perturbation function $H: \mathbb{I} \rightarrow \mathbb{K}^{n \times n}$ as well as structured perturbations of the form

$$
E \dot{x}=A x+B \Delta(C x), t \in \mathbb{I},
$$

where $B: \mathbb{I} \rightarrow \mathbb{K}^{n \times m}$ and $C \in C: \mathbb{I} \rightarrow \mathbb{K}^{q \times n}$ are given matrix functions, restricting the structure of the perturbation and $\Delta: L_{p}\left(0, \infty ; \mathbb{K}^{m}\right) \rightarrow L_{p}\left(0, \infty ; \mathbb{K}^{q}\right)$ is an unknown perturbation operator. The exponential stability radius is then defined to be the largest bound $R$ such that the exponential stability is preserved for all perturbations $H$ or $\Delta$, respectively, of norm strictly less than $R$.

To obtain formulas for the exponential stability radius, we assume that (19) is exponentially stable, i.e., there exist constants $L>0, \gamma>0$ such that $\left\|\Phi_{0}(t, s) P(s)\right\| \leq L e^{-\gamma(t-s)}$, for $t \geq s \geq 0$, and that it is robustly index one in the sense that, supplied with a bounded projection $Q$, the matrix functions $G^{-1}$ and $-Q G^{-1} \widehat{A}$ are essentially bounded on $\mathbb{I}$.

To extend the tractability index concept to the perturbed system (26), we assume that the perturbation operator $\Delta \in \mathscr{L}\left(L_{p}\left(0, \infty ; \mathbb{K}^{q}\right), L_{p}\left(0, \infty ; \mathbb{K}^{m}\right)\right)$ is causal which is defined as follows. For $T \in \mathbb{I}$, the truncation operator $\pi_{T}$ at $T$ on $L_{p}(0, \infty ; X)$ is defined via

$$
\pi_{T}(u):=\left\{\begin{array}{l}
u, t \in[0, T] \\
0, t>T
\end{array}\right.
$$

An operator $\mathbb{P} \in \mathscr{L}\left(L_{p}(0, \infty ; X), L_{p}(0, \infty ; Y)\right)$ then is said to be causal, if $\pi_{T} \mathbb{P} \pi_{T}=\pi_{T} \mathbb{P}$ for every $T \in \mathbb{I}$. Let the linear operator $\widetilde{G} \in \mathscr{L}\left(L_{p}^{\text {loc }}\left(0, \infty ; \mathbb{K}^{n}\right), L_{p}^{\text {loc }}\left(0, \infty ; \mathbb{K}^{n}\right)\right)$ be defined via

$$
(\widetilde{G} z)=(E-\widehat{A} Q) z-B \Delta(C Q z), t \in \mathbb{I} .
$$

If for every $T>0$, the operator $\widetilde{G}$ restricted to $L_{p}\left(0, T ; \mathbb{K}^{n}\right)$ is invertible and the inverse operator $\widetilde{G}^{-1}$ is bounded, then we say the perturbed DAE (26) is of index one (in a generalized sense). Then we can employ the concept of mild solution.

Definition 8. We say that the initial value problem for the perturbed system (26) with initial condition

$$
P\left(t_{0}\right)\left(x\left(t_{0}\right)-x_{0}\right)=0,
$$

admits a mild solution if there exists $x \in L_{p}^{l o c}\left(t_{0}, \infty ; \mathbb{K}^{n}\right)$ satisfying

$$
x(t)=\Phi\left(t, t_{0}\right) P\left(t_{0}\right) x_{0}+\int_{t_{0}}^{t} \Phi(t, \rho) P G^{-1} B \Delta\left([C x(\cdot)]_{t_{0}}\right)(\rho) d \rho+Q G^{-1} B \Delta\left([C x(\cdot)]_{t_{0}}\right)(t)
$$

for $t \geq t_{0}$, where

$$
[C x(\cdot)]_{t_{0}}= \begin{cases}0, & t \in\left[0, t_{0}\right) \\ C(t) x(t), & t \in\left[t_{0}, \infty\right)\end{cases}
$$


The existence and uniqueness of mild solutions is given by the following result.

Theorem 5. [31] Consider the initial value problem (26)-(27). If (26) is of index at most one, then it admits a unique mild solution $x \in L_{p}^{\text {loc }}\left(t_{0}, \infty ; \mathbb{K}^{n}\right)$ with absolutely continuous $z=$ Px for all $t_{0} \in \mathbb{I}, x_{0} \in \mathbb{K}^{n}$. Furthermore, for an arbitrary $T>0$, there exists a constant $L_{1}$ such that pointwise

$$
\|P(t) x(t)\| \leq L_{1}\left\|P\left(t_{0}\right) x_{0}\right\| \text { for all } t \in\left[t_{0}, T\right] .
$$

With (26), we associate the perturbation operator

$$
\mathbb{L}_{t_{0}} z=C \int_{t_{0}}^{t} \Phi(t, \rho) P G^{-1} B(\rho) z(\rho) d \rho+C Q G^{-1} B z,
$$

which is defined for all $t \geq t_{0} \geq 0, z \in L_{p}\left(0, \infty ; \mathbb{K}^{m}\right)$. For exponentially stable robust index one systems this operator is linear, bounded, and monotonically non-increasing with respect to $t$, i.e.,

$$
\left\|\mathbb{L}_{t_{0}}\right\| \geq\left\|\mathbb{L}_{t_{1}}\right\| \quad \text { for all } t_{1} \geq t_{0} \geq 0
$$

and for all $t \in \mathbb{I}$ the bound

$$
\left\|\mathbb{L}_{t}\right\| \leq \frac{L}{\gamma}\left\|P G^{-1}\right\|_{\infty}\|B\|_{\infty}\|C\|_{\infty}+\left\|C Q G^{-1} B\right\|_{\infty}
$$

holds.

Denoting by $x\left(t ; t_{0}, x_{0}\right)$ the solution of (26) with initial condition $x\left(t_{0}\right)=x_{0}$, the trivial solution of (26) is said to be globally $L_{p}-$ stable, if there exist a constant $L_{2}>0$ such that

$$
\left\|x\left(\cdot ; t_{0}, x_{0}\right)\right\|_{L_{p}\left(t_{0}, \infty ; \mathbb{K}^{n}\right)} \leq L_{2}\left\|P\left(t_{0}\right) x_{0}\right\|_{\mathbb{K}^{n}},
$$

for all $t \geq t_{0}, x_{0} \in \mathbb{K}^{n}$, see [31]. Note that this kind of stability notion is equivalent to the concept of output stability, see [53] for various stability concepts in the ODE case.

We then have the following definition of stability radii for time-varying DAEs.

Definition 9. If system (19) is exponentially stable and robustly index one, then the complex/real structured stability radii of (19) subject to linear, dynamic and causal perturbations as in (26), are defined by

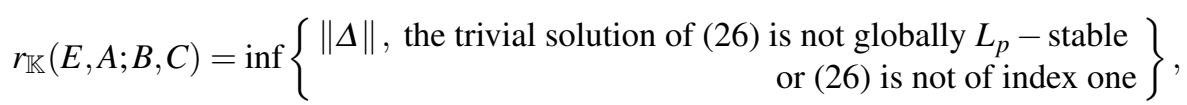

where $\mathbb{K}=\mathbb{C}$ or $\mathbb{K}=\mathbb{R}$, respectively.

In [31] the following formulas for the stability radii were derived.

Theorem 6. [31] If system (19) is exponentially stable and robustly index one, then

$$
r_{\mathbb{K}}(E, A ; B, C)=\min \left\{\lim _{t_{0} \rightarrow \infty}\left\|\mathbb{L}_{t_{0}}\right\|^{-1},\left(\operatorname{ess} \sup _{t \in \mathbb{I}}\left\|C Q G^{-1} B(t)\right\|\right)^{-1}\right\} .
$$

This implies the following corollary.

Corollary 1. If system (19) is exponentially stable and robustly index one and the data $(E, A ; B, C)$ are real, then

$$
r_{\mathbb{C}}(E, A ; B, C)=r_{\mathbb{R}}(E, A ; B, C) .
$$

As special case we obtain the formula (12) for the complex stability radius of time-invariant systems.

Corollary 2. Let $E, A, B, C$ be time-invariant, let the system (19) be index one and exponentially stable. If $p=2$, i.e., for the space $L_{2}$ of square integrable functions

$$
r_{\mathbb{C}}(E, A ; B, C)=\left\|\mathbb{L}_{0}\right\|^{-1}=\left(\sup _{\omega \in i \mathbb{R}}\left\|C(\omega E-A)^{-1} B\right\|\right)^{-1} .
$$


Note that the formula obtained in Corollary 2 is just a special case of that in Theorem 1 when only $A$ is subject to perturbations and in this case the complex stability radius with respect to dynamic perturbations and that to static perturbations are equal. This results generalizes that for the ODE case in [49].

Example 5. [31, Sect. 5.1] Consider a DAE in semi-explicit form

$$
E=\left[\begin{array}{rr}
I_{r} & 0 \\
0 & 0
\end{array}\right], A=\left[\begin{array}{ll}
A_{11} & A_{12} \\
A_{21} & A_{22}
\end{array}\right]
$$

with analogous partitioning. The index one assumption means that $A_{22}(t)$ is invertible almost everywhere in $\mathbb{I}$. One gets $Q=\operatorname{diag}\left(0, I_{n-r}\right)$,

$$
G=\left[\begin{array}{c}
I_{r}-A_{12} \\
0-A_{22}
\end{array}\right], \Phi(t, s)=\left[\begin{array}{c}
\widehat{\Phi}(t, s) \\
-A_{22}^{-1} A_{21} \widehat{\Phi}(t, s)
\end{array}\right],
$$

where $\widehat{\Phi}(t, s)$ is the fundamental solution operator of the underlying ordinary differential equation $\dot{y}=$ $\left(A_{11}-A_{12} A_{22}^{-1} A_{21}\right) y$, which is assumed to be exponentially stable. The assumption that the system is robustly index means the essential boundedness of $A_{22}^{-1}, A_{22}^{-1} A_{21}$ and $A_{12} A_{22}^{-1}$. Partitioning the restriction matrices $B, C$ as $B=\left[\begin{array}{ll}B_{1}^{T} & B_{2}^{T}\end{array}\right]^{T}, C=\left[\begin{array}{ll}C_{1} & C_{2}\end{array}\right]$, analogously, we obtain

$$
\left(\mathbb{L}_{t_{0}} u\right)(t)=\left(C_{1}-C_{2} A_{22}^{-1} A_{21}\right) \int_{t_{0}}^{t} \widehat{\Phi}(t, \rho)\left(B_{1}(\rho)-A_{12} A_{22}^{-1} B_{2}(\rho)\right) u(\rho) d \rho-C_{2} A_{22}^{-1} B_{2} u(t),
$$

and by Theorem 6 we have

$$
r_{\mathbb{K}}(E, A ; B, C)=\min \left\{\lim _{t_{0} \rightarrow \infty}\left\|\mathbb{L}_{t_{0}}\right\|^{-1},\left(\operatorname{ess} \sup _{t \in \mathbb{I}}\left\|C_{2} A_{22}^{-1} B_{2}(t)\right\|\right)^{-1}\right\} .
$$

In summary, we have seen in this section that in the index one case the exponential stability radii in the linear time-varying case are similar to the ones in the linear time-invariant case.

A similar analysis can be performed in principle for the asymptotic stability, by studying the Lyapunov exponents under perturbations, see the next section. Unfortunately, however, in contrast to the Bohl exponents, the Lyapunov exponents themselves may be very sensitive to small changes in the data.

\subsection{Dependence of stability radii on the data}

The robustness analysis of the Bohl exponent was extended from ODEs in [43] to DAEs in [18]. In the context of deriving numerical methods for the numerical computation of Bohl and Lyapunov exponents for linear time-varying DAEs, see $[62,63,65]$ the robustness of these exponents under perturbations was studied and the concept of admissible perturbations was to extended to the variable coefficient case. In this section we summarize these results and study how the stability radii depend on the data.

To see that the Bohl exponent is robust under sufficiently small index one preserving perturbations, we consider the perturbed equation

$$
E \frac{d}{d t}(P x)=(\widehat{A}+H) x
$$

and assume that

$$
\sup _{t \in \mathbb{I}}\|H(t)\|<\left(\sup _{t \in \mathbb{I}}\left\|Q G^{-1}(t)\right\|\right)^{-1} .
$$

Note that this condition implies the inequality $\sup _{t \in \mathbb{I}}\left\|Q G^{-1} H(t)\right\|<1$, which is essential in the analysis.

Theorem 7. [18] Suppose that system (19) is robustly index one and satisfies (32) and suppose further that for any $\varepsilon>0$ there exists $\delta=\delta(\varepsilon)>0$ such that 


$$
\limsup _{s, t-s \rightarrow \infty} \frac{1}{t-s} \int_{s}^{t}\left\|P G^{-1} H(\tau)\right\| d \tau<\delta
$$

then

$$
k_{B}(E, A+H)<k_{B}(E, A)+\varepsilon .
$$

As a consequence of Theorem 7, the exponential stability is preserved under all sufficiently small perturbations $H$.

Remark 7. The robustness analysis of the Bohl exponent is extendable to the case of general perturbations arising in both coefficients of (19). It is clear that additional assumptions on the perturbation structure and/or the smoothness of the admissible perturbations are necessary in this case. The same can be said for the analysis of Bohl exponents for general higher-index DAEs and for nonlinear perturbations, see [2].

We now discuss how the structured stability radius of (25) depends on the perturbation $H$ and the restriction matrices $B, C$. To this end, we first establish the asymptotic behavior of the norm of the input-output operator defined in (28).

Theorem 8. [18] Suppose that system (19 is exponentially stable, robustly index one and satisfies (32) and suppose, in addition, that the perturbation function $H$ satisfies

$$
\lim _{t \rightarrow \infty}\|H(t)\|=0
$$

Then the operator $\mathbb{L}_{t}$ defined in (28) satisfies

$$
\lim _{t \rightarrow \infty}\left\|\mathbb{L}_{t}\right\|=\lim _{t \rightarrow \infty}\left\|\widetilde{\mathbb{L}}_{t}\right\|
$$

By invoking Theorem 6, we obtain sufficient conditions for a sequence of perturbations $H_{k}$ under which the structured stability radius of the perturbed systems converges to that of the unperturbed system.

Theorem 9. [18] Suppose that system (19) is exponentially stable, robustly index one and satisfies (32). Let $\left\{H_{k}(\cdot)\right\}_{k=1}^{\infty}$ be a sequence of measurable matrix functions and suppose that $\sup _{t \in \mathbb{I}}\left\|H_{k}(t)\right\|<$ $\left(\sup _{t \in \mathbb{I}}\left\|Q G^{-1}(t)\right\|\right)^{-1}$ for all $k=1,2, \ldots$ and $\lim _{k \rightarrow \infty} \sup _{t \in \mathbb{I}}\left\|H_{k}(t)\right\|=0$. Then

$$
\lim _{k \rightarrow \infty} r_{\mathbb{K}}\left(E, A+H_{k} ; B, C\right)=r_{\mathbb{K}}(E, A ; B, C) .
$$

Theorem 9 implies that the stability radius for the system (19) depends continuously on the entries of the coefficient matrix function $A$ and as a consequence of Theorem 2, we get the following corollary.

Corollary 3. Let E, A, B, C be constant matrices, let system (19) be of index one and exponentially stable. Furthermore, assume that the sequence of time-varying perturbation $\left\{H_{k}\right\}_{k=1}^{\infty}$ fulfills the conditions of Theorem 9. Then, for the Euclidean norm, one has

$$
\lim _{k \rightarrow \infty} r_{\mathbb{C}}\left(E, A+H_{k} ; B, C\right)=\left(\sup _{w \in i \mathbb{R}}\left\|C(w E-A)^{-1} B\right\|\right)^{-1} .
$$

To illustrate this result, in [18, Example 5.13], a numerical example is given which shows that the complex stability radius of a linear time-invariant system under time-varying perturbations is computable by that for the corresponding time-invariant DAE.

Finally, one also obtains that the structured stability radius of (19) depends continuously on the restriction matrices $B$ and $C$ as in the time-invariant case.

Theorem 10. [18] Suppose that system (19) is exponentially stable, robustly index one and satisfies (32). Let $B_{k}$ and $C_{k}$ be two sequences of measurable and essentially bounded matrix functions satisfying

$$
\lim _{k \rightarrow \infty} \operatorname{ess} \sup _{t \in \mathbb{I}}\left\|B_{k}(t)-B(t)\right\|=0, \quad \lim _{k \rightarrow \infty} \operatorname{ess} \sup _{t \in \mathbb{I}}\left\|C_{k}(t)-C(t)\right\|=0
$$

then,

$$
\lim _{k \rightarrow \infty} r_{\mathbb{K}}\left(E, A ; B_{k}, C_{k}\right)=r_{\mathbb{K}}(E, A ; B, C)
$$


We stress once more that, since the dynamics of DAEs is constrained and the index one property should be preserved, only weaker results hold for the continuity of the stability radius and more restrictive assumptions are required than in the ODE case. Furthermore, for time-varying DAEs, we have to restrict the analysis to perturbations in $A$ only, see (25) and (26), simply because the study of perturbations associated with the leading term $E$ is still mainly an open problem.

In order to study the robustness of Lyapunov exponents, we consider the specially perturbed system

$$
[E+F] \dot{x}=[A+H] x, \quad t \in \mathbb{I},
$$

where $F=\left[\begin{array}{c}F_{1} \\ 0\end{array}\right]$ and $H=\left[\begin{array}{c}H_{1} \\ H_{2}\end{array}\right]$, and where $F_{1}$ and $H_{1}, H_{2}$ are assumed to have the same order of smoothness as $E_{1}$ and $A_{1}, A_{2}$, respectively. Perturbations of this structure are called admissible, generalizing the concept for the constant coefficient DAEs studied in [15].

The DAE (22) is said to be robustly strangeness-free if it stays strangeness-free under all sufficiently small admissible perturbations and it is easy to see that this property holds if and only if the matrix function $\hat{E}$ is boundedly invertible.

If we assume that (22) is already given in the form (23), then the perturbed DAE has the form

$$
\begin{aligned}
\left(E_{11}+F_{11}\right) \frac{d}{d t} \tilde{x}_{1}+\left(E_{12}+F_{12}\right) \frac{d}{d t} \tilde{x}_{2} & =\left(A_{11}+H_{11}\right) \tilde{x}_{1}+\left(A_{12}+H_{12}\right) \tilde{x}_{2} \\
0 & =H_{21} \tilde{x}_{1}+\left(A_{22}+H_{22}\right) \tilde{x}_{2} .
\end{aligned}
$$

In the following we restrict ourselves to robustly strangeness-free DAE systems under admissible perturbations.

Definition 10. The upper Lyapunov exponents $\lambda_{1}^{u} \geq \ldots \geq \lambda_{d}^{u}$ of (22) are said to be stable if for any $\varepsilon>0$, there exists $\delta>0$ such that the conditions $\sup _{t}\|F(t)\|<\delta$, $\sup _{t}\|H(t)\|<\delta$, and $\sup _{t}\left\|\dot{H}_{2}(t)\right\|<\delta$ on the perturbations imply that the perturbed DAE system (33) is strangeness-free and

$$
\left|\lambda_{i}^{u}-\gamma_{i}^{u}\right|<\varepsilon, \quad \text { for all } i=1,2, \ldots, d,
$$

where the $\gamma_{i}^{u}$ are the ordered upper Lyapunov exponents of (33).

The boundedness condition on $\dot{H}_{2}$, which is obviously satisfied in the time-invariant setting [15], is an extra condition and it seems to be somehow unusual. However, the DAE (24) shows the necessity.

As in the ODE case, see [1,23], to have stability of the Lyapunov spectrum, one needs the property of integral separation, i.e., for the columns of the minimal fundamental solution matrix $\Phi$ of (22) there exist constants $c_{1}>0$ and $c_{2}>0$ such that

$$
\frac{\left\|X(t) e_{i}\right\|}{\left\|X(s) e_{i}\right\|} \cdot \frac{\left\|X(s) e_{i+1}\right\|}{\left\|X(t) e_{i+1}\right\|} \geq c_{2} e^{c_{1}(t-s)}
$$

for all $t, s$ with $t \geq s \geq 0$ and $i=1, \ldots, d-1$. Then we have the following sufficient conditions for the stability of the upper Lyapunov exponents of (22).

Theorem 11. [62] Consider the DAE (22) in the form (23). Suppose that the matrix $\hat{E}$ is boundedly invertible and that $E_{11}^{-1} A_{11}, A_{12} A_{22}^{-1}$ and the derivative of $A_{22}$ are bounded on $\mathbb{I}$. Then, the upper Lyapunov exponents of (22) are distinct and stable if and only if the system has the integral separation property.

This shows that if perturbations are performed in $E$ as well, then the perturbation analysis of time-varying DAEs requires more restrictive conditions than in the time-invariant case. However, for some classes of structured problems and/or structured perturbation, parts of these conditions can be relaxed.

If the perturbation block $H_{21}$ disappears, i.e., if $H$ and $A$ have the same block triangular structure, then for example the restrictive conditions on the derivatives in Definition 10 and Theorem $11 \mathrm{can}$ be omitted. Similar situations happen in the case that $E_{12}=F_{12}=0$ as discussed in [62, Section 3.2] and the case of perturbations in $A$ only as in (25). 
In [62] another stability concept, the Sacker-Sell spectrum has been extended to linear DAEs with variable coefficients. It is shown also shown that unlike the Lyapunov spectrum, the Sacker-Sell spectrum is robust in the sense that it is stable without requiring integral separation.

This means that for general strangeness-free time-varying systems, the exponential stability is robust, but the asymptotic stability is not. However, this remark does not apply to time-invariant systems, for which the two stability notions are equivalent.

Remark 8. The robustness analysis for linear DAEs of index higher than one and under general perturbations is essentially an open problem. The same is true for the distances to the other important control properties such as controllability and observability. The robustness of these concepts for linear DAEs with variable coefficients presents a major challenge.

\section{Discussion}

In this paper we have surveyed recent results on the robustness of asymptotic and exponential stability for linear time-invariant and time-varying DAEs. We have analyzed robust stability and its distance measures, the real or complex structured stability radius and presented formulas and various properties of the stability radii. We have seen that the robustness analysis for DAEs is much more complicated than that for ODEs. In general, results already known for ODEs now hold for DAEs only under extra assumptions, mainly restricting the set of admissible perturbations. DAE aspects also give rise to new robustness and distance problems. While for time-invariant DAEs, most of the robustness and distance problems are well understood, many problems for time-varying DAEs are still open. These and robustness analysis for general nonlinear and/or high-index DAEs and time-delay DAEs are interesting and challenging topics for future work.

\section{References}

1. L. Ya. Adrianova. Introduction to linear systems of differential equations, Trans. Math. Monographs, Vol. 146, AMS, Providence, RI, 1995.

2. T. Berger. Bohl exponent for time-varying linear differential-algebraic equations. Int. Journal of Control, 2012 (to appear).

3. P. Bohl. Über differentialungleichungen. J.f. d. Reine und Angew. Math., 144:284-313, 1913.

4. S. Boyd and V. Balakrishnan. A Regularity Result for the Singular Values of a Transfer Matrix and a Quadratically Convergent Algorithm for Computing its $L_{\infty}$-norm. Systems Control Letters, 15:1-7, 1990.

5. M. Bracke. On stability radii of parametrized linear differential-algebraic systems. Ph.D. Thesis, University of Kaiserslautern, 2000.

6. N.A. Bruinsma and M. Steinbuch. A fast algorithm to compute the $H_{\infty}$ of a transfer function matrix. Systems Control Letters, 14:287-293, 1990.

7. K. E. Brenan, S. L. Campbell, and L. R. Petzold. Numerical Solution of Initial-Value Problems in Differential Algebraic Equations. SIAM Publications, Philadelphia, PA, 2nd edition, 1996.

8. A. Bunse-Gerstner, R. Byers, V. Mehrmann and N.K. Nichols, Feedback Design for Regularizing Descriptor Systems. Linear Algebra Appl. 299:119-151, 1999.

9. A. Bunse-Gerstner, V. Mehrmann and N.K. Nichols, Regularization of descriptor dystems by derivative and proportional state feedback. SIAM J. Matrix Anal. Appl., 13:46-67, 1992.

10. A. Bunse-Gerstner, V. Mehrmann and N.K. Nichols, Regularization of descriptor dystems by output feedback. IEEE Trans. Automatic Control, 39:1742-1748, 1994.

11. J. Burke, A.S. Lewis, and M.L. Overton. Optimization and pseudospectra, with applications to robust stability. SIAM J. Matrix Anal. Appl., 25:80-104, 2003.

12. R. Byers. A Bisection Method for Measuring the Distance of a Stable Matrix to the Unstable Matrices. SIAM J. Sci. and Stat. Comput., 9:875-881, 1988.

13. R. Byers. The descriptor controllability radius. Systems and Networks: Mathematical Theory and Application, Proceedings of MTNS '93, Akademie Verlag, Berlin, 85-88, 1994.

14. R. Byers, C. He, and V. Mehrmann. Where is the nearest non-regular pencil? Lin. Alg. Appl., 285:81-105, 1998.

15. R. Byers and N.K. Nichols. On the stability radius of a generalized state-space system. Lin. Alg. Appl., 188-189:113-134, 1993.

16. S. L. Campbell. Linearization of DAE's along trajectories. Z. Angew. Math. Phys., 46:70-84, 1995. 
17. S. L. Campbell, N. K. Nichols, and W. J. Terrell. Duality, observability, and controllability for linear time-varying descriptor systems. Circ. Syst. Signal Process., 10:455-470, 1991.

18. C.J. Chyan, N.H. Du, and V.H. Linh. On data-dependence of exponential stability and the stability radii for linear timevarying differential-algebraic systems. J. Diff. Equations, 245:2078-2102, 2008.

19. N.D. Cong and H. Nam. Lyapunov's inequality for linear differential algebraic equation. Acta Math. Vietnam, 28:73-88, 2003.

20. N.D. Cong and H. Nam. Lyapunov regularity of linear differential algebraic equations of index 1. Acta Math. Vietnam, 29:1-21, 2004.

21. L. Dai. Singular Control Systems. Lecture Notes in Control and Information Science, Springer-Verlag, 1989.

22. J.L. Daleckii and M.G. Krein. Stability of solutions of differential equations in Banach spaces. American Mathematical Society, Providence, RI, 1974.

23. L. Dieci and E. S. Van Vleck. Lyapunov spectral intervals: theory and computation, SIAM J. Numer. Anal., 40:516-542, 2002.

24. M. Dmitriev and G. Kurina. Singular perturbation in control problems. Automation and Remote Control, 67:1-43, 2006.

25. V. Dragan. The asymptotic behavior of the stability radius for a singularly perturbed linear system. Int. J. Robust and Nonlin. Control, 8:817-829, 1998.

26. V. Dragan and A. Halanay Stabilization of Linear Systems. Birkhäuser, Boston, 1999.

27. N.H. Du. Stability radii of differential-algebraic equations with structured perturbations. Systems Control Letters, 57:546$553,2008$.

28. N.H. Du, D.T. Lien, and V.H. Linh. Complex stability radii for implicit discrete-time systems. Vietnam J. Math., 31:475$488,2003$.

29. N.H. Du and V.H. Linh Implicit-system approach to the robust stability for a class of singularly perturbed linear systems. Systems Control Letters, 54:33-41, 2005.

30. N.H. Du and V.H. Linh. Robust stability of implicit linear systems containing a small parameter in the leading term. IMA J. Math. Cont. Inf., 23:67-84, 2006.

31. N.H. Du and V.H. Linh. Stability radii for linear time-varying differential-algebraic equations with respect to dynamic perturbations. J. Diff. Equations, 230:579-599, 2006.

32. N.H. Du, D.D. Thuan, and N.C. Liem. Stability radius of implicit dynamic equations with constant coefficients on time scales. Systems Control Letters, 60:596-603, 2011.

33. E. Eich-Soellner and C. Führer. Numerical Methods in Multibody Systems. Teubner Verlag, Stuttgart, Germany, 1998.

34. M.A. Freitag and A. Spence. A Newton-based method for the calculation of the distance to instability. Linear Alg. Appl., 435(12): 3189-3205, 2011.

35. F.R. Gantmacher, Theory of Matrices. Vol. 2, Chelsea, New York, 1959.

36. G. H. Golub and C. F. Van Loan. Matrix Computations. The Johns Hopkins University Press, Baltimore, MD, 3rd edition, 1996.

37. E. Griepentrog and R. März. Differential-Algebraic Equations and their Numerical Treatment. Teubner Verlag, Leipzig, Germany, 1986.

38. M. Gu, E. Mengi, M. L. Overton, J. Xia and J. Zhu, Fast Methods for Estimating the Distance to Uncontrollability SIAM J. Matrix Anal. Appl. 28:477-502, 2006.

39. M. Gürbüzbalaban and M. L. Overton, Some Regularity Results for the Pseudospectral Abscissa and Pseudospectral Radius of a Matrix. SIAM J. Optimization 22:281-285, 2012.

40. N. Guglielmi and M. L. Overton, Fast Algorithms for the Approximation of the Pseudospectral Abscissa and Pseudospectral Radius of a Matrix SIAM J. Matrix Anal. Appl. 32:1166-1192, 2011.

41. E. Hairer and G. Wanner. Solving Ordinary Differential Equations II: Stiff and Differential-Algebraic Problems. SpringerVerlag, Berlin, Germany, 2nd edition, 1996.

42. C. He, G.A. Watson. An algorithm for computing the distance to instability. SIAM J. Matrix Anal. Appl., 20:101-116, 1999.

43. D. Hinrichsen, A. Ilchmann, A.J. Pritchard. Robustness of stability of time-varying linear systems. J. Differential Equations, 82:219-250, 1989.

44. D. Hinrichsen and B. Kelb. Spectral value sets: a graphical tool for robustness analysis. Systems Control Letters, 21(2), 127-136, 1993.

45. D. Hinrichsen, B. Kelb, and A. Linnemann. An algorithm for the computation of the complex stability radius. Automatica, 25:771-775, 1989.

46. D. Hinrichsen and A.J. Pritchard, Stability radii of linear systems. Systems Control Letters, 7:1-10, 1986.

47. D. Hinrichsen and A.J. Pritchard, Stability radii for structured perturbations and the algebraic Riccati equations. Systems Control Letters, 8:105-113, 1986.

48. D. Hinrichsen and A.J. Pritchard A note on some difference between real and complex stability radii. Systems Control Letters, 14:401-408, 1990.

49. D. Hinrichsen, A.J. Pritchard. Destabilization by output feedback. Differential Integral Equations, 5:357-386, 1992.

50. D. Hinrichsen and A.J. Pritchard. On spectral variations under bounded real matrix perturbations Numer. Math., 60:509524, 1992.

51. D. Hinrichsen and A. J. Pritchard. Mathematical Systems Theory I. Modelling, State Space Analysis, Stability and Robustness. Springer-Verlag, New York, NY, 2005.

52. G. Hu. Robustness measures for linear time-invariant time-delay systems. Ph.D. Thesis, University of Toronto, 2001. 
53. A. Ilchmann, I.M.Y. Mareels. On stability radii of slowly time-varying systems. In: Advances in mathematical system theory, Boston, Birkhäuser, 55-75, 2001.

54. B. Jacob. A formula for the stability radius of time-varying systems. J. Differential Equations, 142:167-187, 1998.

55. P. Kokotovic, H.K Khalil, and J. O'Reilly Singular Perturbation Method in Control: Analysis and Design. Academic, New York, 1986.

56. P. Kunkel and V. Mehrmann. Differential-Algebraic Equations. Analysis and Numerical Solution. EMS Publishing House, Zürich, Switzerland, 2006.

57. P. Kunkel, V. Mehrmann, W. Rath, and J. Weickert. A new software package for linear differential-algebraic equations. SIAM J. Sci. Comput., 18:115-138, 1997.

58. S. Lam. Real robustness radii and performance limitations of LTI control systems. Ph.D. Thesis, University of Toronto, 2011.

59. S. Lam and E.J. Davison. Real controllability radius of high-order, descriptor, and time-delay LTI systems. in: Proceedings of the 18th IFAC World Congress, Milano (Italy), August 28 -September 2, 2011, 6 pages, 2011.

60. R. Lamour, R. März, and C. Tischendorf. Differential-Algebraic Equations: A Projector Based Analysis. Springer, 2013 (to appear).

61. L. Lee, C-H. Fang, J-G. Hsieh, Exact unidirectional perturbation bounds for robustness of uncertain generalized statespace systems: continuous-time cases. Automatica J. IFAC, 33:1923-1927, 1997.

62. V.H. Linh and V. Mehrmann. Lyapunov, Bohl and Sacker-Sell spectral intervals for differential-algebraic equations. $J$. Dynamics Diff. Eq., 21:153-194, 2009.

63. V.H. Linh and V. Mehrmann. Approximation of spectral intervals and associated leading directions for linear differentialalgebraic systems via smooth singular value decompositions. SIAM J. Numer. Anal., 49:1810-1835, 2011.

64. V.H. Linh and V. Mehrmann. Spectral analysis for linear differential-algebraic systems. In Proceedings of the 8th AIMS International Conference on Dynamical Systems, Differential Equations and Applications, Dresden, May 24-28, 2010, DCDS Supplement, 991-1000, 2011.

65. V.H. Linh, V. Mehrmann, and E. Van Vleck. $Q R$ methods and error analysis for computing lyapunov and sacker-sell spectral intervals for linear differential-algebraic equations. Adv. Comput. Math., 35:281-322, 2011.

66. A. M. Lyapunov. The general problem of the stability of motion. Translated by A. T. Fuller from E. Davaux's French translation (1907) of the 1892 Russian original. Internat. J. Control, pages 521-790, 1992.

67. R.M.M. Mattheij and P.M.E.J. Wijckmans. Sensitivity of solutions of linear DAE to perturbations of the system matrices. Numer. Alg., 19:159-171, 1998.

68. V. Mehrmann, The Autonomous Linear Quadratic Control Problem: Theory and Numerical Solution. LeCTURE Notes in CONTROL ANd Information SCIEnCES, Vol. 163, Springer Verlag, Heidelberg, Juli 1991.

69. V. Mehrmann, Index concepts for differential-algebraic equations. PREPRINT 2012-03, Institut für Mathematik, TU Berlin, 2012. url: http://www.math.tu-berlin.de/preprints/.

70. V. Mehrmann and T. Stykel, Descriptor systems: A general mathematical framework for modelling, simulation and control. at-Automatisierungstechnik, 54(8):405-415, 2006.

71. E. Mengi. Measures for robust stability and controllability. Ph.D. Thesis, University of New York, 2006.

72. E. Mengi and M. L. Overton, Algorithms for the Computation of the Pseudospectral Radius and the Numerical Radius of a Matrix IMA J. Numer. Analysis, 25: 48-669, 2005.

73. M. Motscha. Algorithm to compute the complex stability radius. Int. Journal of Control, 48:2417-2428, 1988.

74. L. Qiu, B. Bernhardsson, A. Rantzer, E.J. Davison, P.M. Young, and J.C. Doyle A formula for computation of the real stability radius. Automatica, 31:879-890, 1995.

75. L. Qiu, and E.J. Davison The stability robustness of generalized eigenvalues. IEEE Transactions on Automatic Control, 37:886-891, 1992.

76. P. J. Rabier and W. C. Rheinboldt. Theoretical and Numerical Analysis of Differential-Algebraic Equations, volume VIII of Handbook of Num. Analysis. Elsevier Publications, Amsterdam, The Netherlands, 2002.

77. R. Riaza. Differential-algebraic systems. Analytical aspects and circuit applications. World Scientific Publishing Co. Pte. Ltd., Hackensack, NJ., 2008.

78. R.J. Sacker and G.R. Sell. A spectral theory for linear differential systems. J. Diff. Equations, 27:320-358, 1978.

79. N.K. Son and D. Hinrichsen. Robust stability of positive continuous time systems. Numer. Funct. Anal. Optim., 17:649659, 1996.

80. N.K. Son and D.D. Thuan. The structured distance to non-surjectivity and its application to calculating the controllability radius of descriptor systems. J. Math. Anal. Applic., 388:272-281, 2012.

81. J. Sreedhar, P. Van Dooren, and A. Tits. A fast algorithm to compute the real structured stability radius. Int. Series of Numer. Math., 121:219-230, 1996.

82. H. Tidefelt. Differential-algebraic equations and matrix-valued singular perturbation. Ph.D. Thesis, Linkping University, 2009.

83. L.N. Trefethen and M. Embree. Spectra and Pseudospectra: The Behavior of Nonnormal Matrices and Operators. Princeton University Press, 2005.

84. C.F. Van Loan. How near is a stable matrix to an unstable matrix? Contemp. Math., 47:465-477, 1985. 